\title{
Commentary: Intermittent Fasting and Akkermansia Muciniphila Potentiate the Antitumor Efficacy of FOLFOX in Colon Cancer
}

\author{
Junhong Su, Henri Braat, Auke Verhaar and Maikel Peppelenbosch* \\ Department of Gastroenterology and Hepatology, Erasmus MC-University Medical Center Rotterdam, Rotterdam, Netherlands
}

Keywords: intermittent fasting, the gut microbiome, humans, coloretal cancer, translational medicine

\section{A Commentary on}

Akkermansia Muciniphila Potentiates the Antitumor Efficacy of FOLFOX in Colon Cancer by Hou, X., Zhang, P., Du, H., Chu, W., Sun, R., Qin, S., Tian, Y., Zhang, Z and Xu, F. (2021). Front Pharmacol. 12:725583. doi:10.3389/fphar.2021.725583

Edited by:

Thomas Brzozowski,

Jagiellonian University Medical

College, Poland

Reviewed by:

Shahrbanoo Keshavarz Azizi Raftar,

Pasteur Institute of Iran, Iran

Sun Tian,

Carbon Logic Biotech (HK) Ltd., China

Martín Marcial-Coba,

Pontificia Universidad Católica del

Ecuador, Ecuador

${ }^{*}$ Correspondence:

Maikel Peppelenbosch

m.peppelenbosch@erasmusmc.n

Specialty section: This article was submitted to Translational Pharmacology,

a section of the journal

Frontiers in Pharmacology

Received: 24 December 2021

Accepted: 17 January 2022

Published: 11 February 2022

Citation:

Su J, Braat H, Verhaar A and

Peppelenbosch M (2022)

Commentary: Intermittent Fasting and Akkermansia Muciniphila Potentiate the Antitumor Efficacy of FOLFOX in

Colon Cancer.

Front. Pharmacol. 13:843133.

doi: 10.3389/fphar.2022.843133
Akkermansia muciniphila, a commensal bacterium inhabiting the human intestinal tract, attracts substantial attention as a potential beneficial organism. Being the major producer of propionate in the human intestine, it is endowed with anti-inflammatory properties and linked to improved outcomes in a variety of settings, including the prevention of colorectal cancer (Bian et al., 2019; Su et al., 2020) and metabolic disorders (Plovier et al., 2017). This notion is confirmed by the study Huo et al. (2021), recently published in Frontiers in Pharmacology. In this study the author elegantly show that a pharmacotherapeutical modulatory effect of A. muciniphila in a colon cancer xenograft model (CCXM), enhancing FOLFOX efficiency against experimental colorectal cancer. The authors found that the antitumor effects of FOLFOX in CCXM mice were associated with increased abundance of A. muciniphila. Oral supplementation with A. muciniphila remarkably enhanced FOLFOX efficiency against colon cancer. This study provides hopes for patients with colorectal cancer that by modulating A. muciniphila levels also clinical outcomes of FOLFOX treatment can be enhanced. For establishing such effects, however, it is essential to come up with credible strategies that are capable of increasing intestinal levels of $A$. municiphila.

In this context it is important to note that the abundance of A. muciniphila is significantly decreased not only in colorectal cancer mice, but also in patients with histologically-confirmed adenoma or colorectal cancer (Wang et al., 2020). Likewise, oral supplementation with A. muciniphila reduces colitis-associated tumorigenesis in mice through attenuating DNA damage, increasing tumour cell apoptosis and reducing abnormal proliferation, apparently via increasing the number of $\mathrm{CD} 8^{+}$ cytotoxic T lymphocytes (Wang et al., 2020). More impressively, even a membrane protein from A. muciniphila by itself can exerts effects on colon cancer, similar to those observed when using the pasteurised entire bacterium (Wang et al., 2020). These findings are interpreted as to reflect synergic antitumor effects of this commensal bacterium, which apparently involves at least the production of the relevant bacterial metabolites (i.e., short chain fatty acids) and downstream pathways (Louis et al., 2014) as well as with the effects elicited by the membrane components of this pathway. The emerging insight into the mechanisms mediating A. muciniphila effects in colorectal cancer further prompts the development of strategies aimed at increasing its intestinal levels in the patients involved.

Unfortunately, the earlier studies by Hou et al. (2021) and Wang et al. (2020) do not address this point directly and further work is necessary to exploit the potential of this bacterium in increasing the efficacy of. FOLFOX. Importantly, we recently conducted a study in volunteers either submitting 
A

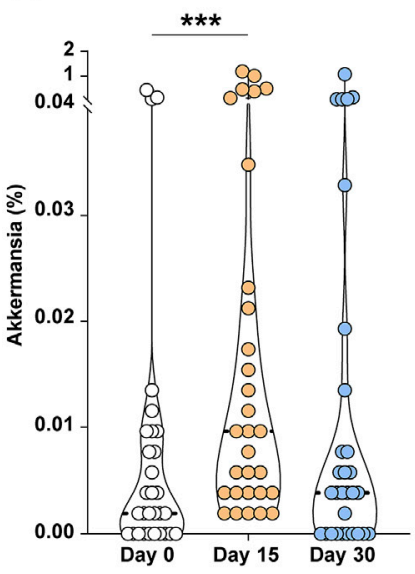

B

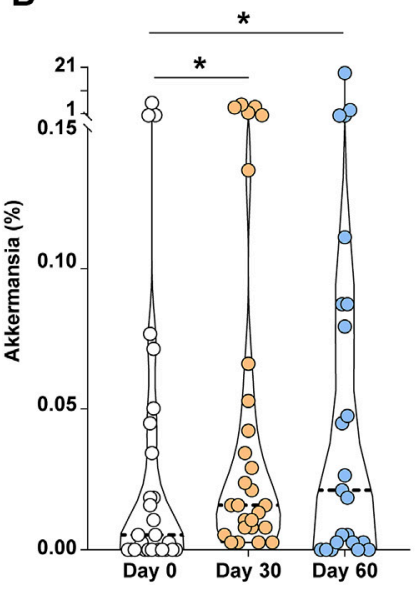

C

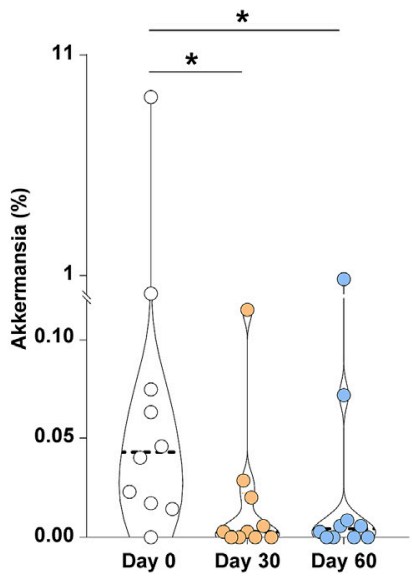

D

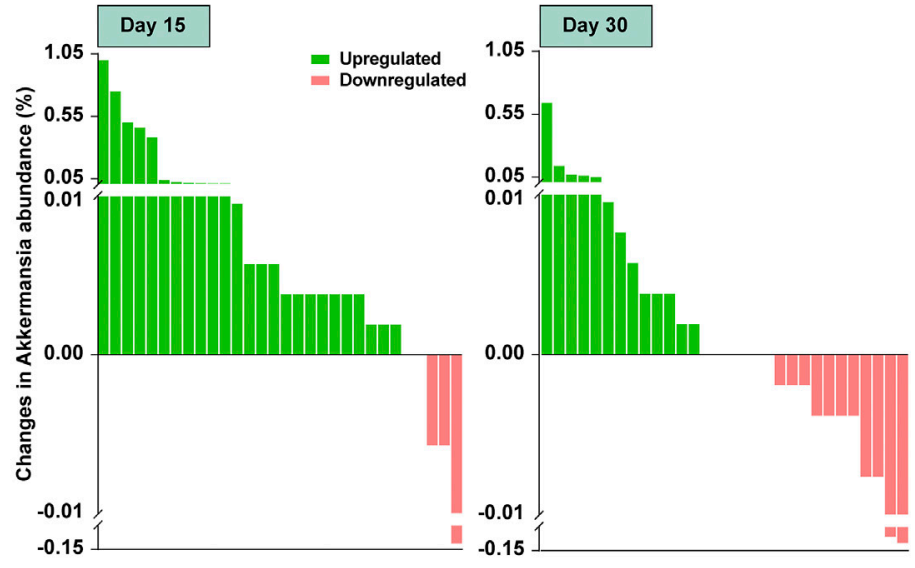

E

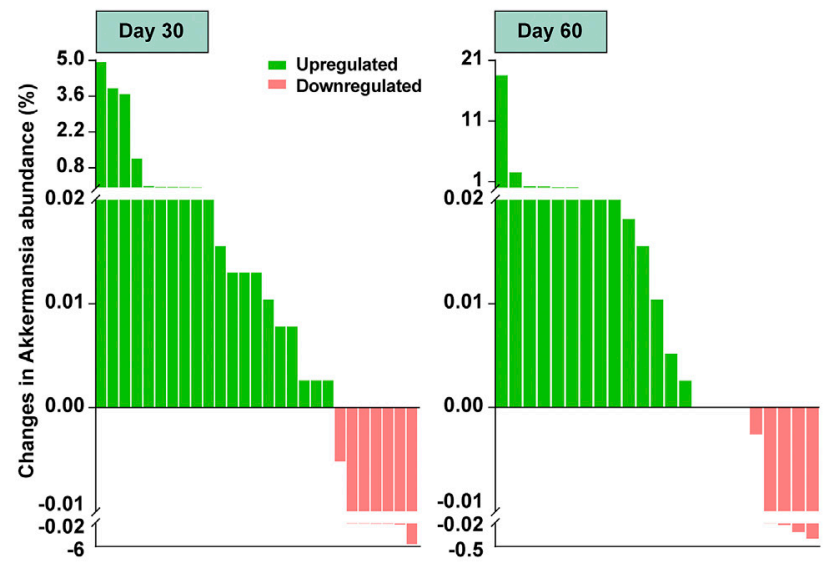

$\mathbf{F}$

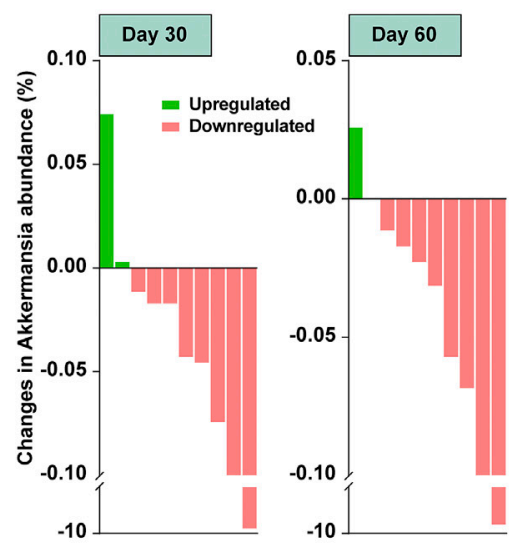

FIGURE 1 | Intermittent fasting upregulated the Akkermansia municiphila species abundance in fecal samples obtained in two independent adult cohorts (for details, see reference 6). (A) Results showing the dynamics of mean abundance of the bacterium from a cohort of young adult males undergoing intermittent fasting. Fecal samples were collected before (day $0 ; n=30$ ), during (day $15 ; n=30$ ) and at the end of intermittent fasting (day $30 ; n=30$ ). ${ }^{\star \star *} p<.001$ by Wilcoxon-singed rank test. (B) The dynamics of mean abundance of the bacterium from a middle-aged cohort undergoing intermittent fasting. Fecal samples were collected before (day $0 ; n=27$ ), at the end of (day 30;n=27), and 30 days following the cessation of intermittent fasting (day $60 ; n=23$ ). ${ }^{*} p<0.05$ by Wilcoxon-singed rank test. (C) The 
FIGURE 1 | dynamics of mean abundance of the bacterium from middle-aged unfasted volunteers. Fecal samples were collected at the same time point as in Figure 1B ( $n=10$ ). ${ }^{*} p<0.05$ by Wilcoxon-singed rank test. (D) Change in $A$. municiphila at day 15 (left) or day 30 (right) was calculated for each individual in the young cohort by subtracting the relative abundance observed before fasting from that of during or after fasting. As a result, 83.3 percent (25/30) of volunteers displayed upregulated bacterium abundance at day 15 and 43.3 percent at day 30. (E) Change in A. municiphila at day 30 (left) or day 60 (right) for each individual in the middle-aged cohort was calculated as above. The bacterium was upregulated in 74.1 percent of volunteers (20/27) at day 30 and 60.9 percent (14/23) at day 60 . (F) Change in $A$. municiphila at day 30 (left) or day 60 (right) was calculated for each unfasted individual as above. The bacterium abundance was increased in only 20 percent of volunteers (2/10) at day 30 and 10 percent $(1 / 10)$ at day 60 in this group. In the present study, $16 \mathrm{~S}$ rRNA sequencing was applied to calculate the abundance of $A$. municiphila at each time point.

themselves a month of intermittent fasting (involving approximately $16 \mathrm{~h}$ of absence of food intake per day) or served as non-fasting controls and characterised the microbiome in these volunteers (Su et al., 2021). Strikingly, among the various effects intermittent fasting exerted on the microbiome we also observed that $A$. municiphila levels were strongly upregulated in the fasting volunteers (Figures 1A,B), but inversely changed in unfasted volunteers (Figure 1C). Furthermore, A. municiphila was increased in more than $83 \%$ individuals in the young cohort (Figure 1D) and $74 \%$ in the middle-aged cohort (Figure 1E), possibly as a consequence of the competitive advantage this organism obtains in the absence of food as it can degrade intestinal mucins. However, upregulated in no more than $20 \%$ individuals in unfasted

\section{REFERENCES}

Bian, X., Wu, W., Yang, L., Lv, L., Wang, Q., Li, Y., et al. (2019). Administration of Akkermansia Muciniphila Ameliorates Dextran Sulfate Sodium-Induced Ulcerative Colitis in Mice. Front. Microbiol. 10, 2259. doi:10.3389/fmicb. 2019.02259

Hou, X., Zhang, P., Du, H., Chu, W., Sun, R., Qin, S., et al. (2021). Akkermansia Muciniphila Potentiates the Antitumor Efficacy of FOLFOX in Colon Cancer. Front. Pharmacol. 12, 725583. doi:10.3389/fphar.2021.725583

Louis, P., Hold, G. L., and Flint, H. J. (2014). The Gut Microbiota, Bacterial Metabolites and Colorectal Cancer. Nat. Rev. Microbiol. 12, 661-672. doi:10. 1038/nrmicro3344

Plovier, H., Everard, A., Druart, C., Depommier, C., Van Hul, M., Geurts, L., et al. (2017). A Purified Membrane Protein from Akkermansia Muciniphila or the Pasteurized Bacterium Improves Metabolism in Obese and Diabetic Mice. Nat. Med. 23, 107-113. doi:10.1038/nm.4236

Su, J., Braat, H., and Peppelenbosch, M. P. (2020). Gut Microbiota-Derived Propionate Production May Explain Beneficial Effects of Intermittent Fasting in Experimental Colitis. J. Crohns Colitis 15 (6), 1081-1082. doi:10. 1093/ecco-jcc/jjaa248

Su, J., Wang, Y., Zhang, X., Ma, M., Xie, Z., Pan, Q., et al. (2021). Remodeling of the Gut Microbiome during Ramadan-Associated Intermittent Fasting. Am. J. Clin. Nutr. 113, 1332-1342. doi:10.1093/ajcn/nqaa388

Wang, L., Tang, L., Feng, Y., Zhao, S., Han, M., Zhang, C., et al. (2020). A Purified Membrane Protein from Akkermansia Muciniphila or the Pasteurised group (Figure 1F). These data would fit well recent data that fasting per se decreases the propensity to contract colorectal cancer (Weng et al., 2020). Thus in conjunction with the data presented by Hou et al. (2021) it would thus be rational to propose that intermittent fasting for patients who are taking medications such as FOLFOX or patients at risk for colorectal cancer (e.g., patients with inflammatory bowel with a history of neoplasms) would benefit from intermittent fasting.

\section{AUTHOR CONTRIBUTIONS}

All authors listed have made a substantial, direct, and intellectual contribution to the work and approved it for publication.

Bacterium Blunts Colitis Associated Tumourigenesis by Modulation of CD8+ T Cells in Mice. Gut 69, 1988-1997. doi:10.1136/gutjnl-2019-320105

Weng, M. L., Chen, W. K., Chen, X. Y., Lu, H., Sun, Z. R., Yu, Q., et al. (2020). Fasting Inhibits Aerobic Glycolysis and Proliferation in Colorectal Cancer via the Fdft1-Mediated AKT/mTOR/HIF1 a Pathway Suppression. Nat. Commun. 11, 1869. doi:10.1038/s41467-020-15795-8

Conflict of Interest: The authors declare that the research was conducted in the absence of any commercial or financial relationships that could be construed as a potential conflict of interest.

The handling editor declared a past co-authorship with one of the authors MP.

Publisher's Note: All claims expressed in this article are solely those of the authors and do not necessarily represent those of their affiliated organizations, or those of the publisher, the editors and the reviewers. Any product that may be evaluated in this article, or claim that may be made by its manufacturer, is not guaranteed or endorsed by the publisher.

Copyright (c) 2022 Su, Braat, Verhaar and Peppelenbosch. This is an open-access article distributed under the terms of the Creative Commons Attribution License (CC $B Y)$. The use, distribution or reproduction in other forums is permitted, provided the original author(s) and the copyright owner(s) are credited and that the original publication in this journal is cited, in accordance with accepted academic practice. No use, distribution or reproduction is permitted which does not comply with these terms. 